\title{
Senior-Boichis syndrome
}

INSERM

\section{Source}

INSERM. (1999). Orphanet: an online rare disease and orphan drug data base. SeniorBoichis syndrome. ORPHA:84081

Boichis syndrome consists of the association of congenital nephronophthisis leading to renal failure, and hepatic fibrosis. It has been described in five members of one family, two of whom died from renal failure. The association of Boichis syndrome with tapetoretinal degeneration and intellectual deficit has also been reported in one family: the so-called Senior-Boichis syndrome could be in fact the same entity, and was later reported in a 12 year-old child. 